\title{
Amplify-and-Forward Cooperative OFDM with Multiple-Relays: Performance Analysis and Relay Selection Methods
}

\author{
Yanwu Ding, Senior Member, IEEE, and Murat Uysal, Senior Member, IEEE
}

\begin{abstract}
In this paper, we investigate a cooperative system with multiple relays and amplify-and-forward relaying over frequency-selective channels. To extract the available multipath diversity, we employ orthogonal frequency division multiplexing (OFDM) with precoding. Through the derivation of pairwise error probability (PEP), we demonstrate that PEP is not a simple exponential function of the signal-to-noise ratio (SNR), but it includes a term that involves some power of logarithm of the SNR. If that term is ignored, the diversity order is given by the summation of the channel length in the direct link and the minimum of channel lengths in each relaying link confirmed by simulation results. Based on the PEP expression, we also propose two relay selection strategies; one is on a per-subcarrier basis and the other is on an all-subcarrier basis. Our simulation results indicate that both strategies result in performance improvements although the per-subcarrier method performs better.
\end{abstract}

Index Terms-Amplify-and-forward relaying, OFDM, pairwise error probability, precoding, relay selection.

\section{INTRODUCTION}

C OOPERATIVE diversity [1] has emerged as a powerful technique to reap the benefits of multiple-input multipleoutput (MIMO) communications in a wireless scenario with single-antenna terminals. By creating a virtual antenna array among the spatially distributed nodes through relaying, this technique realizes the advantages of MIMO communications in a distributed fashion. For example, the cooperation protocol proposed in Laneman et al.'s pioneering work [1] effectively realizes receive diversity (RD). Multihop relaying can be considered a special case of the RD protocol where the destination node only switches on during the relaying phase [2]. Other cooperation protocols such as TD (transmit diversity) and STD (simplified transmit diversity) have also been proposed in the literature [2]. These basic cooperation protocols can be used with either amplify-and-forward (AF) or decode-and-forward (DF) relaying. In DF relaying, the relay node fully decodes, re-encodes, and retransmits the source node's message. In AF relaying, the relay retransmits a scaled version of the received signal without any attempt to decode it.

Related Literature and Contributions of This Work: Pioneering works on the topic of cooperative diversity have been

Manuscript received April 19, 2008; revised December 15, 2008 and May 20, 2009; accepted July 7, 2009. The associate editor coordinating the review of this letter and approving it for publication was A. Chockalingam.

Y. Ding is with the Department of Electrical Engineering and Computer Science, Wichita State University, 1845 Fairmount, Wichita, KS 67260 (email: yanwu.ding@wichita.edu).

M. Uysal is with the Department of Electrical and Computer Engineering, University of Waterloo, Waterloo, ON, Canada, N2L3G1 (e-mail: muysal@ece.uwaterloo.ca).

The work of M. Uysal is supported in part by a Natural Sciences and Engineering Research Council of Canada (NSERC) Special Research Opportunity Grant (SROPJ305821-05).

Digital Object Identifier 10.1109/TWC.2009.081642 built upon the assumption of frequency-flat fading channels. However, in broadband communication systems, the transmission bandwidth is larger than the coherence bandwidth of the channel which results in a frequency-selective channel. A widely used approach to overcome the degrading effects of frequency-selectivity is OFDM which has been recently applied to cooperative communications. Particularly, OFDM-based multihop relaying has been studied by several authors (see e.g., [3]-[6] and the references therein). The integration of RD, TD, and STD protocols with OFDM has also attracted some attention in the literature [7]-[9]. However, these works are mainly limited to single-relay scenarios.

In contrast to most earlier work, this paper considers a multi-relay scenario and investigates the performance limits of a cooperative precoded OFDM system for RD cooperation protocol. Specifically, we derive a PEP for the precoded cooperative OFDM system under consideration and demonstrate the asymptotical diversity order. Based on the PEP expression, we further propose two relay selection methods which work on a per-subcarrier basis and an all-subcarrier basis respectively. Finally, we present Monte-Carlo simulations to demonstrate the achievable diversity gains in the practical SNR range and the impact of proposed relay selection methods on the error rate performance.

Notation: Bold upper- and lower-case letters denote respectively matrices and column vectors (e.g., A, a). $(\cdot)^{T}$ and $(\cdot)^{H}$ denote transpose and conjugate-transpose operations respectively. A vector $\mathbf{s}$ of length $P$ is expressed as $\mathbf{s}=[\mathbf{s}(1), \mathbf{s}(2), \cdots, \mathbf{s}(P)]$, with $\|\mathbf{s}\|$ standing for its 2-norm. $\mathbf{X}(i, j)$ denotes the $(i, j)^{\text {th }}$ element of matrix $\mathbf{X}$. The $i^{\text {th }}$ diagonal element in diagonal matrix $\mathbf{D}$ is denoted by $\mathbf{D}(i)$. $\mathbf{I}_{K}$ denotes a $K \times K$ identity matrix and $\mathbf{0}$ stands for an all-zero matrix of appropriate dimensions. $Q$ represents the $P \times P$ FFT (Fast Fourier Transform) matrix. $\mathbf{V}_{\mathrm{AB}}$ denotes a $P \times\left(L_{\mathrm{AB}}+1\right)$ matrix with elements given by $\mathbf{V}_{\mathrm{AB}}(k, m)=\exp (-j 2 \pi(k-1)(m-1) / P), 1 \leq k \leq$ $P, 1 \leq m \leq\left(L_{\mathrm{AB}}+1\right) . \mathbb{E}[\cdot]$ is the expectation operator. Notation $f(x) \triangleq O(g(x)), f(x), g(x)>0$ denotes the existence of a positive constant $c$ such that $f(x) \leq c g(x)$ when $x$ is large.

\section{System Model}

We consider a cooperative wireless communication scenario where the source node $\mathrm{S}$ transmits information to the destination node $\mathrm{D}$ with the assistance of $N$ relay nodes $\mathrm{R}_{1}, \mathrm{R}_{2}, \cdots, \mathrm{R}_{N}$. All nodes are equipped with a single antenna and operate in half-duplex mode. Any linear modulation technique such as PSK (phase shift keying) or QAM (quadrature amplitude modulation) can be used. The cooperation is based 
on the RD protocol with $\mathrm{AF}$ relaying: In the broadcasting phase, the source node transmits to the destination and the relay nodes. In the relaying phase, the relay nodes take turns to forward a scaled noisy version of the signals received from the source.

To take into account the effect of relay geometry, we consider an aggregate channel model which consists of both long-term path loss and short-term fading effects. The path loss is proportional to $d^{-\alpha}$ where $d$ is the propagation distance and $\alpha$ is the path loss coefficient. Normalizing the path loss in the source-to-destination $(\mathrm{S} \rightarrow \mathrm{D})$ link to be unity, the relative gains from source-to- $n^{\text {th }}$ relay ( $\mathrm{S}$ $\left.\rightarrow \mathrm{R}_{n}\right)$ and from the $n^{\text {th }}$ relay-to-destination $\left(\mathrm{R}_{n} \rightarrow \mathrm{D}\right)$ links are defined, respectively, as $G_{\mathrm{SR}_{n}}=\left(d_{\mathrm{SD}} / d_{\mathrm{SR}_{n}}\right)^{\alpha}$ and $G_{\mathrm{R}_{n} \mathrm{D}}=\left(d_{\mathrm{SD}} / d_{\mathrm{R}_{n} \mathrm{D}}\right)^{\alpha}, n=1,2, \cdots, N$ [2]. They can be further related to each other by $G_{\mathrm{SR}_{n}}^{-2 / \alpha}+G_{\mathrm{R}_{n} \mathrm{D}}^{-2 / \alpha}-$ $2 G_{\mathrm{SR}_{n}}^{-1 / \alpha} G_{\mathrm{R}_{n} \mathrm{D}}^{-1 / \alpha} \cos \theta_{n}=1$ through the law of cosines, where $\theta_{n}$ is the angle between links $\mathrm{S} \rightarrow \mathrm{R}_{n}$ and $\mathrm{R}_{n} \rightarrow \mathrm{D}$. The ratio $G_{\mathrm{SR}_{n}} / G_{\mathrm{R}_{n} \mathrm{D}}$ in $\mathrm{dB}$ reflects the effect of relay location. The more negative this ratio in $\mathrm{dB}$, the closer that particular relay is placed to the destination terminal. The underlying channels are modeled as quasi-static frequency-selective Rayleigh fading with a uniform delay profile. The channel impulse responses (CIRs) for $\mathrm{S} \rightarrow \mathrm{R}_{n}, \mathrm{R}_{n} \rightarrow \mathrm{D}$ and $\mathrm{S} \rightarrow \mathrm{D}$ links are given by $\mathbf{h}_{\mathrm{SR}_{n}}=\left[\mathbf{h}_{\mathrm{SR}_{n}}(1), \mathbf{h}_{\mathrm{SR}_{n}}(2), \cdots, \mathbf{h}_{\mathrm{SR}_{n}}\left(L_{\mathrm{SR}_{n}}+1\right)\right]$, $\mathbf{h}_{\mathrm{R}_{n} \mathrm{D}}=\left[\mathbf{h}_{\mathrm{R}_{n} \mathrm{D}}(1), \mathbf{h}_{\mathrm{R}_{n} \mathrm{D}}(2), \cdots, \mathbf{h}_{\mathrm{R}_{n} \mathrm{D}}\left(L_{\mathrm{R}_{n} \mathrm{D}}+1\right)\right]$, and $\mathbf{h}_{\mathrm{SD}}=\left[\mathbf{h}_{\mathrm{SD}}(1), \mathbf{h}_{\mathrm{SD}}(2), \cdots, \mathbf{h}_{\mathrm{SD}}\left(L_{\mathrm{SD}}+1\right)\right]$. The entries of random vectors $\mathbf{h}_{\mathrm{SR}_{n}}, \mathbf{h}_{\mathrm{R}_{n} \mathrm{D}}$, and $\mathbf{h}_{\mathrm{SD}}$ are independent identically distributed (i.i.d) zero mean Gaussian random variables with variances of $1 /\left(L_{\mathrm{SR}_{n}}+1\right), 1 /\left(L_{\mathrm{R}_{n} \mathrm{D}}+1\right)$, and $1 /\left(L_{\mathrm{SD}}+1\right)$ respectively. The CIRs remain constant over a period of one block transmission and vary independently from block to block.

We consider an OFDM relay system in which a linear precoder [10] is used to extract the maximum diversity gain. Let $\mathbf{s}$ denote the information symbol block of length $P$. The precoded data block is expressed as $\mathbf{x}=\Theta \mathbf{s}$ where $\Theta$ is the precoding matrix satisfying power constraint $\operatorname{tr}\left(\Theta \Theta^{H}\right)=P$, where $P$ is the total number of subcarriers. To avoid inter block interference, a cyclic prefix of length $L=\max \left(L_{\mathrm{SD}}, L_{\mathrm{SR}_{n}}, L_{\mathrm{R}_{n} \mathrm{D}}\right)$ is added between adjacent information blocks. Subcarriers are divided into $M$ groups in order to reduce the decoding complexity and simplify the precoder design. Each group contains $L+1$ equally spaced sub-carriers. The precoder $\Theta$ can be expressed as $\Theta=$ $\sum_{k=1}^{M} \boldsymbol{\Psi}_{k}^{T} \boldsymbol{\Phi} \boldsymbol{\Psi}_{k}[10]$, where $\boldsymbol{\Psi}_{k}=\mathbf{I}_{P}\left(i_{k, \ell},:\right)$ is a $(L+1) \times P$ permutation matrix obtained from the rows of identity matrix of size $P$, i.e., $\left\{i_{k, \ell}=(\ell-1) M+k, \ell=1, \cdots, L+1\right\}$, and $\boldsymbol{\Phi}$ is a matrix of size $(L+1) \times(L+1)$ that performs precoding within one group.

Since the FFT operation at the relay nodes is cancelled by the IFFT (inverse FFT) operation in the relaying phase, it is reasonable to assume that FFT processing takes place only at the destination, but not at the relays, to reduce the complexity. After removing the cyclic prefix, the received data block at the $n^{\text {th }}$ relay node is given by $\mathbf{r}_{\mathrm{R}_{n}}=\sqrt{G_{\mathrm{SR}_{n}} E} \mathbf{H}_{\mathrm{SR}_{n}} \mathbf{Q}^{H} \mathbf{x}+$ $\mathbf{n}_{\mathbf{R}_{n}}, \quad n=1,2, \cdots, N$, where $E$ is the average power per transmitting symbol, $\mathbf{H}_{\mathrm{SR}_{n}}$ is a circulant channel matrix with entries of $\mathbf{H}_{\mathrm{SR}_{n}}(i, j)=\left(\mathbf{h}_{\mathrm{SR}_{n}}(i-j) \bmod P\right), i, j=$ $1,2, \cdots, P$, and $\mathbf{n}_{\mathrm{R}_{n}}, n=1,2, \cdots, N$ denotes the zero mean white Gaussian noise vector (with covariance matrix of $N_{0} \mathbf{I}_{P}$ ) at the $n^{\text {th }}$ relay node. To ensure that the power budget is not violated, each relay node normalizes the respective received vector $\mathbf{r}_{\mathrm{R}_{n}}$ by a factor of $\sqrt{\mathbb{E}\left[\left\|\mathbf{r}_{\mathrm{R}_{n}}\right\|^{2}\right] / P}=\sqrt{G_{\mathrm{SR}_{n}} E+N_{0}}$ and then forwards the resulting signal to the destination. The received signals at the destination during the broadcasting and relaying phases are therefore given by

$$
\begin{aligned}
\mathbf{r}_{\mathrm{D}_{0}} & =\sqrt{E} \mathbf{Q} \mathbf{H}_{\mathrm{SD}} \mathbf{Q}^{H} \mathbf{x}+\mathbf{n}_{0} \\
\mathbf{r}_{\mathrm{D}_{n}} & =A_{n} \sqrt{G_{\mathrm{SR}_{n}} E} \mathbf{Q} \mathbf{H}_{\mathrm{R}_{n} \mathrm{D}} \mathbf{H}_{\mathrm{SR}_{n}} \mathbf{Q}^{H} \mathbf{x}+A_{n} \mathbf{H}_{\mathrm{R}_{n} \mathrm{D}} \mathbf{n}_{\mathrm{R}_{n}}+\mathbf{n}_{n}
\end{aligned}
$$

where $A_{n}=\sqrt{G_{\mathrm{R}_{n} \mathrm{D}} E /\left(G_{\mathrm{SR}_{n}} E+N_{0}\right)}$, and $\mathbf{n}_{q}, q=$ $0,1, \cdots, N$, are Gaussian noise vectors at the destination with zero mean and covariance matrix of $N_{0} \mathbf{I}_{P}$. Here, $\mathbf{H}_{\mathrm{SD}}$ and $\mathbf{H}_{\mathrm{SR}_{n}}$ are circulant channel matrices with their $(i, j)^{\text {th }}$ entry being $\mathbf{H}_{\mathrm{SD}}(i, j)=\left(\mathbf{h}_{\mathrm{SD}}(i-j) \bmod P\right)$ and $\mathbf{H}_{\mathrm{R}_{n} \mathrm{D}}(i, j)=$ $\left.\mathbf{h}_{\mathrm{R}_{n} \mathrm{D}}(i-j) \bmod P\right), i, j=1,2, \cdots, P$ respectively.

Now, let $\mathbf{D}_{\mathrm{AB}}(p)=\sum_{k=1}^{L_{\mathrm{AB}}+1} \mathbf{h}_{\mathrm{AB}}(k) \exp (-j 2 \pi(k-1)(p-$ 1) $/ P), \quad p=1,2, \cdots, P$, denote the frequency response evaluated at the FFT grid for the $\mathrm{A} \rightarrow \mathrm{B}$ link with channel length $L_{\mathrm{AB}}+1$. We can rewrite (1) as

$$
\begin{aligned}
& \mathbf{r}_{\mathrm{D}_{0}}=\sqrt{E} \mathbf{D}_{\mathrm{SD} \mathbf{x}+\mathbf{n}_{0}} \\
& \mathbf{r}_{\mathrm{D}_{n}}=A_{n} \sqrt{G_{\mathrm{SR}_{n}} E} \mathbf{D}_{\mathrm{R}_{n} \mathrm{D}} \mathbf{D}_{\mathrm{SR}_{n}} \mathbf{x}+\widetilde{\mathbf{n}}_{n}, n=1,2, \cdots, N
\end{aligned}
$$

where the noise vector $\widetilde{\mathbf{n}}_{n}=A_{n} \mathbf{H}_{\mathrm{R}_{n} \mathrm{D}} \mathbf{n}_{\mathrm{R}_{n}}+\mathbf{n}_{n}$ is conditionally (conditioned on $\mathbf{H}_{\mathrm{R}_{n} \mathrm{D}}$ ) zero mean Gaussian distributed with covariance matrix $N_{0} \boldsymbol{\Sigma}_{n}$ and $\boldsymbol{\Sigma}_{n}=A_{n}^{2} \mathbf{D}_{\mathrm{R}_{n} \mathrm{D}} \mathbf{D}_{\mathrm{R}_{n} \mathrm{D}}^{H}+\mathbf{I}_{P}$. After pre-whitening at the destination, the input signals to the maximum likelihood (ML) detector are given by

$$
\begin{gathered}
\mathbf{r}=\operatorname{diag}\left[\sqrt{E} \mathbf{D}_{\mathrm{SD}}, A_{1} \sqrt{G_{\mathrm{SR}_{1}} E} \boldsymbol{\Sigma}_{1}^{-1 / 2} \mathbf{D}_{\mathrm{R}_{1} \mathrm{D}} \mathbf{D}_{\mathrm{SR}_{1}}, \cdots,\right. \\
\left.A_{N} \sqrt{G_{\mathrm{SR}_{N}} E} \boldsymbol{\Sigma}_{N}^{-1 / 2} \mathbf{D}_{\mathrm{R}_{N} \mathrm{D}} \mathbf{D}_{\mathrm{SR}_{N}}\right] \mathbf{x}+\mathbf{n}
\end{gathered}
$$

where noise vector $\mathbf{n}$ in (3) is zero mean white Gaussian with covariance matrix $N_{0} \mathbf{I}_{P}$.

\section{DeRIVATION OF PEP}

For a given channel realization and transmitted symbol block s, conditional PEP denotes the probability of deciding in favor of another block $\mathbf{s}^{\prime}$ and is given by

$$
P_{e}\left(\mathbf{s} \rightarrow \mathbf{s}^{\prime} \mid \mathbf{h}_{\mathrm{SD}}, \mathbf{h}_{\mathrm{SR}_{n}}, \mathbf{h}_{\mathrm{R}_{n} \mathrm{D}}\right)=Q\left(\frac{d\left(\mathbf{s}, \mathbf{s}^{\prime}\right)}{2}\right)
$$

where $d\left(\mathbf{s} \rightarrow \mathbf{s}^{\prime}\right)$ is the Euclidean distance between $\mathbf{s}$ and $\mathbf{s}^{\prime}$ and $Q(x)$ is the Gaussian- $Q$ function [11]. Let error vector $\mathbf{e}=\mathbf{s}-\mathbf{s}^{\prime}$ represent the difference between $\mathbf{s}$ and $\mathbf{s}^{\prime}$. Then, the precoded error vector is $\mathbf{u}=\boldsymbol{\Theta e}$. Therefore, we have

$$
\begin{aligned}
& d^{2}\left(\mathbf{s} \rightarrow \mathbf{s}^{\prime}\right) \\
& =\rho\left(\left\|\mathbf{D}_{\mathrm{SD}} \mathbf{u}\right\|^{2}+\sum_{n=1}^{N}\left\|A_{n} \sqrt{G_{\mathrm{SR}_{n}}} \boldsymbol{\Sigma}_{n}^{-1 / 2} \mathbf{D}_{\mathrm{R}_{n} \mathrm{D}} \mathbf{D}_{\mathrm{SR}_{n}} \mathbf{u}\right\|^{2}\right)
\end{aligned}
$$

where $\rho=E / N_{0}$ denotes SNR. Notice $\mathbf{D}_{\mathrm{AB}} \mathbf{u}=\mathbf{U}_{\mathrm{AB}} \mathbf{h}_{\mathrm{AB}}$, where $\mathbf{D}_{\mathrm{AB}}$ is a diagonal matrix of size $P \times P, \mathbf{U}_{\mathrm{AB}}=$ $\operatorname{diag}(\mathbf{u}) \mathbf{V}_{\mathrm{AB}}$, and $\mathbf{V}_{\mathrm{AB}}$ is of the size of $P \times\left(L_{\mathrm{AB}}+1\right)$ as defined earlier in Notation. Applying Chernoff bound on (4), 
i.e., $Q(x) \leq \frac{1}{2} \exp \left(-\frac{x^{2}}{2}\right)$, we obtain an upper bound for the average PEP as

$$
P_{e}\left(\mathbf{s} \rightarrow \mathbf{s}^{\prime}\right) \leq \frac{1}{2} \mathbb{E}_{\mathbf{h}_{\mathrm{SD}}}\left[\exp \left(\frac{-\rho \mathbf{h}_{\mathrm{SD}}^{H} \mathbf{U}_{\mathrm{SD}}^{H} \mathbf{U}_{\mathrm{SD}} \mathbf{h}_{\mathrm{SD}}}{8}\right)\right] \prod_{n=1}^{N} J_{n}
$$

where

$$
J_{n}=\mathbb{E}_{\mathbf{h}_{\mathrm{SR}_{n}}, \mathbf{h}_{\mathrm{R}_{n} \mathrm{D}}}\left[\exp \left(\frac{-\rho\left\|\sqrt{G_{\mathrm{SR}_{n}}} A_{n} \boldsymbol{\Sigma}^{-1 / 2} \mathbf{D}_{\mathrm{R}_{n} \mathrm{D}} \mathbf{D}_{\mathrm{SR}_{n}} \mathbf{u}\right\|^{2}}{8}\right)\right] .
$$

Following steps in the Appendix, we obtain

$$
\begin{aligned}
P_{e}\left(\mathbf{s} \rightarrow \mathbf{s}^{\prime}\right) & \leq \beta \rho^{-\left(L_{\mathrm{SD}}+1+\sum_{n=1}^{N} k_{n}\right)}(\ln \rho)^{\sum_{n=1}^{N} k_{n}} \\
+ & O\left(\rho^{-\left(L_{\mathrm{SD}}+1+\sum_{n=1}^{N} k_{n}\right)}(\ln \rho)^{\sum_{n=1}^{N}\left(k_{n}-1\right)}\right)
\end{aligned}
$$

where $\beta$ is defined as

$$
\beta=\frac{8^{L_{\mathrm{SD}}+1}(8 \pi)^{\sum_{n=1}^{N} k_{n}}\left(L_{\mathrm{SD}}+1\right)^{L_{\mathrm{SD}}+1} \prod_{n=1}^{N}\left(L_{\mathrm{SR}_{n}}+1\right)^{k_{n}}}{2 \operatorname{det}\left(\mathbf{U}_{\mathrm{SD}}^{H} \mathbf{U}_{\mathrm{SD}}\right)\left(\prod_{n=1}^{N} \varpi_{n}\right)\left(v G_{\mathrm{R}_{n} \mathrm{D}}\right)^{\sum_{n=1}^{N} k_{n}}}
$$

and $k_{n}=\min \left(L_{\mathrm{SR}_{n}}+1, L_{\mathrm{R}_{n} \mathrm{D}}+1\right), v=\left(2^{\frac{1}{L+1}}-1\right) \Delta_{m i n}^{2}$, $\Delta_{\text {min }}$ is the minimum Euclidean distance among the constellation points, and $\varpi_{n}=\operatorname{det}\left(\mathbf{Q}_{n} \mathbf{Q}_{n}^{H}\right)$. Here, matrix $\mathbf{Q}_{n}$ contains the $1^{\text {st }},(M+1)^{\text {th }}, \cdots,\left(M L_{\mathrm{SR}_{n}}+1\right)^{\text {th }}$ rows in $\mathbf{V}_{\mathrm{SR}_{n}}$.

It is observed from (8) that the PEP is not a simple exponential function of the SNR. It involves the term $(\ln \rho)^{\sum_{n=1}^{N} \min \left(L_{\mathrm{SR}_{n}}+1, L_{\mathrm{R}_{n} \mathrm{D}}+1\right)}$ due to the cascaded nature of channels in the relaying links. This is in line with the observation of the term $(\ln \rho)^{N}$ reported in [12], [13] for frequencyflat channels. From (8), we also observe that an asymptotical diversity order of $K=L_{\mathrm{SD}}+1+\sum_{n=1}^{N} \min \left(L_{\mathrm{SR}_{n}}+1, L_{\mathrm{R}_{n} \mathrm{D}}+\right.$ 1 ) is available for the precoded OFDM multi-relay system.

\section{PEP-Based Relay Selection Methods}

So far, we have assumed the participation of all $N$ relay nodes in the relaying phase for the considered multi-relay scenario. As described in Section II, the relay nodes take turns to forward the received signals from the source. A potential alternative transmission scheme is relay selection which yields a higher throughput, because fewer time slots are required to complete the transmission of one block. Most of the current work on relay selection assumes frequency-flat channels with a few exceptions such as [5] and [6] which focus on frequencyselective channels. In [5], Siriwongpairat et al. have considered an OFDM cooperative system with a fixed number of relays in DF relaying and proposed a relay assignment scheme for the RD protocol based on the outage probability. In [6], a multihop scenario has been considered for DF relaying based on the outage probability. In the following, we propose two relay selection methods based on the PEP expression.

The first method is on a per-subcarrier basis in which the "best" relay is chosen for each subcarrier. The second method is on an all-subcarrier basis in which a single relay is selected for all subcarriers. From (4) and (5), we readily check that the PEP depends on the following quantity associated with the $p^{\text {th }}$ subcarrier for the $n^{\text {th }}$ relay, $p=1,2, \cdots, P, n=1,2, \cdots, N$,

$$
\zeta_{n}(p)=\left|A_{n} \sqrt{G_{\mathrm{SR}_{n}}} \boldsymbol{\Sigma}_{n}(p)^{-1 / 2} \mathbf{D}_{\mathrm{R}_{n} \mathrm{D}}(p) \mathbf{D}_{\mathrm{SR}_{n}}(p)\right|^{2}
$$

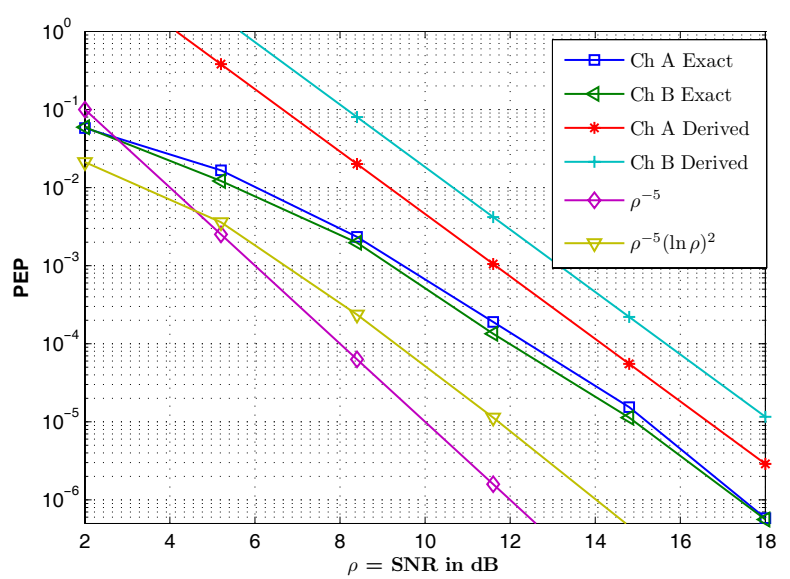

Fig. 1. Comparison of exact and derived PEP.

$$
=\frac{A_{n}^{2} G_{\mathrm{SR}_{n}}\left|\mathbf{D}_{\mathrm{R}_{n} \mathrm{D}}(p) \mathbf{D}_{\mathrm{SR}_{n}}(p)\right|^{2}}{1+A_{n}^{2}\left|\mathbf{D}_{\mathrm{R}_{n} \mathrm{D}}(p)\right|^{2}}
$$

where $\boldsymbol{\Sigma}_{n}(p), \mathbf{D}_{\mathrm{R}_{n} \mathrm{D}}(p)$, and $\mathbf{D}_{\mathrm{SR}_{n}}(p)$ denote the $p^{\text {th }}$ diagonal elements in matrices $\boldsymbol{\Sigma}_{n}, \mathbf{D}_{\mathrm{R}_{n} \mathrm{D}}$, and $\mathbf{D}_{\mathrm{SR}_{n}}$ respectively. Based on (10), we propose two strategies for relay selection:

- Relay Selection Method 1 (RSM-1): For each subcarrier in the relaying phase, we select the relay which provides the largest $\zeta_{n}(p)$ for that subcarrier.

- Relay Selection Method 2 (RSM-2): For each relay, we calculate $\sum_{p=1}^{P} \zeta_{n}(p)$ and choose the relay that provides the highest sum. The chosen relay will be used for all subcarriers in the relaying phase.

In RSM-1, the relays are selected on a per-subcarrier basis, therefore, the number of active relays in the relaying phase can be as high as $N$. On the other hand, in RSM-2, there is only one relay active in the relaying phase. Therefore, RSM-2 has lower operation cost and complexity than RSM-1. However, as demonstrated later, the performance for RSM-2 is not as good as RSM-1.

\section{Numerical Results}

\section{A. Diversity Gain}

To verify the diversity gain predicted by the derived PEP, we calculate the exact PEP by taking the average of (4) (with respect to random channels) through a Monte-Carlo simulation and compare it with (8). We consider a single relay system in which the relay is located at an equal distance to the source and to the destination, i.e., $G_{\mathrm{SR}} / G_{\mathrm{RD}}=0 \mathrm{~dB}, \theta=60^{\circ}$, and assume QPSK modulation. For the convenience of evaluating (4), we set $P=L+1$ where $L=\max \left(L_{\mathrm{SD}}, L_{\mathrm{SR}}, L_{\mathrm{RD}}\right)$. We consider an error event of length 4 with $\mathbf{s}=\frac{1}{\sqrt{2}}\left[\begin{array}{llll}1+j & 1+j & 1+j & 1-j\end{array}\right]^{T}$ and $\mathbf{s}^{\prime}=\frac{1}{\sqrt{2}}[1+j-1+j \quad 1-j-1-j]^{T}$ assuming two channel configurations:

Channel A: $L_{\mathrm{SD}}=2, L_{\mathrm{SR}}=1, L_{\mathrm{RD}}=3$;

Channel B: $L_{\mathrm{SD}}=2, L_{\mathrm{SR}}=3, L_{\mathrm{RD}}=1$.

Fig. 1 shows the comparison of exact and derived PEP. The derived PEP expression is able to precisely reflect the slope of the exact PEP, which quantifies the diversity gain. The discrepancy between the derived and exact PEP is mainly 


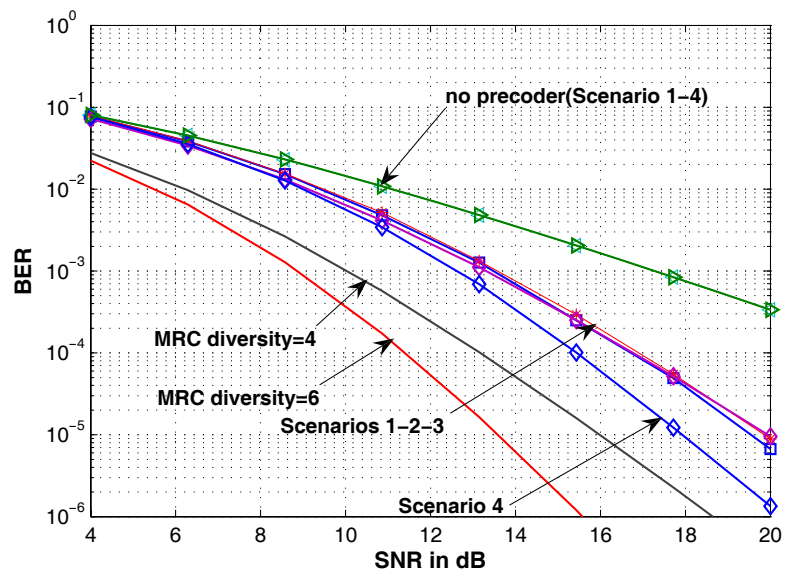

Fig. 2. BER performance of precoded cooperative OFDM with one relay.

due to the deployed Chernoff bound. For the specific channel lengths under consideration, we observe that the PEP behaves in the form of $\rho^{-5}(\ln \rho)^{2}$. We have also included a plot of $\rho^{-5}$ as a benchmark curve to demonstrate the degradation resulting from $(\ln \rho)^{2}$.

\section{B. BER Performance}

In this section, we present Monte-Carlo simulation results to demonstrate the error rate performance of the precoded cooperative OFDM system under consideration. We again assume QPSK modulation, and set $\theta_{n}=60^{\circ}$ for all relays. In order to perform the grouping technique [10], the number of subcarriers is chosen rounded to the nearest integer of $P=64$ depending on the value of $L+1=\max \left(L_{\mathrm{SD}}+1, L_{\mathrm{R}_{n} \mathrm{D}}+\right.$ $\left.1, L_{\mathrm{SR}_{n}}+1\right)$. Specifically, we investigate Scenarios $1-4$ for the single relay system and Scenarios $5-8$ for the two-relay system with the following channel orders:

Scenario 1: $L_{\mathrm{SD}}=1, L_{\mathrm{SR}}=1, L_{\mathrm{RD}}=2$;

Scenario 2: $L_{\mathrm{SD}}=1, L_{\mathrm{SR}}=1, L_{\mathrm{RD}}=1$;

Scenario 3: $L_{\mathrm{SD}}=1, L_{\mathrm{SR}}=2, L_{\mathrm{RD}}=1$;

Scenario 4: $L_{\mathrm{SD}}=2, L_{\mathrm{SR}}=2, L_{\mathrm{RD}}=2$;

Scenario 5: $L_{\mathrm{SD}}=1, L_{\mathrm{SR}_{n}}=1, L_{\mathrm{R}_{n} \mathrm{D}}=2, n=1,2$

Scenario 6: $L_{\mathrm{SD}}=1, L_{\mathrm{SR}_{n}}=1, L_{\mathrm{R}_{n} \mathrm{D}}=1, n=1,2$

Scenario 7: $L_{\mathrm{SD}}=1, L_{\mathrm{SR}_{n}}=2, L_{\mathrm{R}_{n} \mathrm{D}}=1, n=1,2$

Scenario 8: $L_{\mathrm{SD}}=2, L_{\mathrm{SR}_{n}}=2, L_{\mathrm{R}_{n} \mathrm{D}}=2, n=1,2$

As clearly seen from Figs. 2 and 3, without the use of precoding, diversity gain is limited to spatial diversity (which is equal to 2 and 3 for the single relay system and the tworelay system, respectively), and is independent of channel lengths. Through the deployment of precoding, OFDM is able to extract the underlying rich multipath diversity.

For the single relay system, it is observed from Fig. 2 that an identical diversity order is achieved for the Scenarios 1, 2 , and 3 , as predicted by our diversity gain analysis (i.e., minimum of channel lengths over relaying link determines the diversity order of this link). The diversity order increases for Scenario 4, where the channel lengths of both source-to-relay and relay-to-destination links are equal to 3 . The maximum diversity order available for these scenarios is obtained as $L_{\mathrm{SD}}+1+\min \left(L_{\mathrm{SR}}+1, L_{\mathrm{RD}}+1\right)=4$ and 6 , respectively.

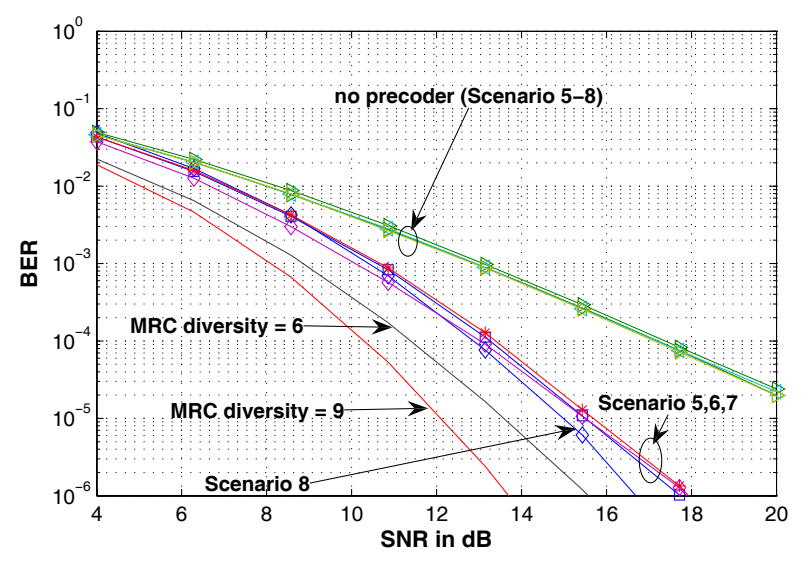

Fig. 3. BER performance of cooperative precoded OFDM with two relays.

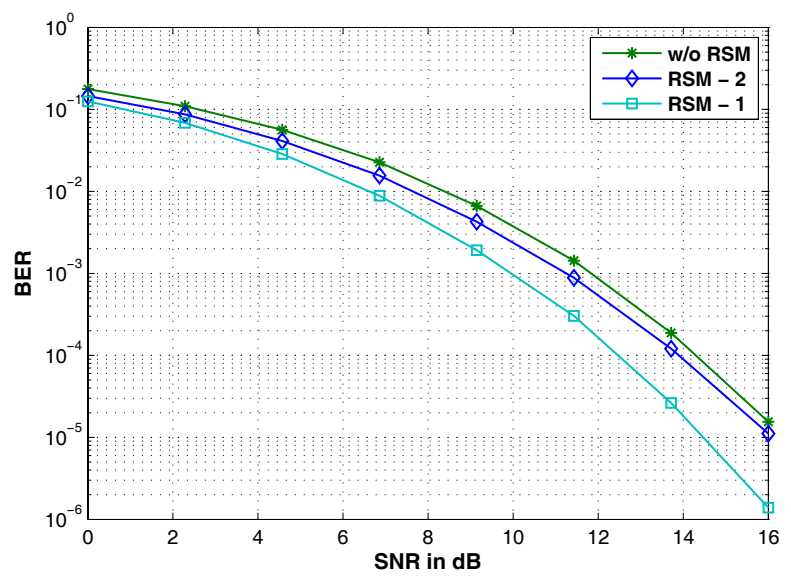

Fig. 4. Performance of relay selection methods for a three-relay system with $G_{\mathrm{SR}_{n}} / G_{\mathrm{R}_{n} \mathrm{D}}=0 \mathrm{~dB}, n=1,2,3$.

However, the cascaded nature of Rayleigh channels in relaying links allows only partial diversity to be observed in the practical SNR range under consideration [14]. This can be observed better through a comparison with the performance of maximum ratio combining (MRC) with 4 and 6 co-located antennas, which is also included in Fig. 2, for benchmarking purpose.

For the two-relay system (i.e., Scenarios 5-8), the maximum diversity orders are calculated as $L_{\mathrm{SD}}+1+\sum_{n=1}^{N} \min \left(L_{\mathrm{SR}_{n}}+\right.$ $\left.1, L_{\mathrm{R}_{n} \mathrm{D}}+1\right)=6$ and 9. As expected, Scenarios 5-7 yield an identical diversity order while in Scenario 8, a higher diversity order is obtained. The performance of MRC schemes with 6 and 9 co-located antennas are also included in Fig. 3 for comparison purposes. In comparison to these point-to-point links, a performance degradation is observed in the two-relay system, similar to that observed in Fig. 2.

\section{Relay Selection Methods}

In Fig. 4, we investigate the performance of relay selection methods RSM-1 and RSM-2 described in Section IV. Subcarrier sorting in the relaying phase is adopted in our schemes [4], [15]. We consider a three-relay system with $L_{\mathrm{SD}}=L_{\mathrm{SR}_{n}}=L_{\mathrm{R}_{n} \mathrm{D}}=1$ and relay locations $G_{\mathrm{SR}_{n}} / G_{\mathrm{R}_{n} \mathrm{D}}=$ 
OdB, $n=1,2,3$. We observe from Fig. 4 that RSM-2 provides about $0.5 \mathrm{~dB}$ performance improvement (at $\mathrm{BER}=10^{-3}$ ) over the original scheme in which all relays participate in the relaying phase. The performance gain climbs up to about $1.5 \mathrm{~dB}$ for RSM-1, where the relay selection is performed on a subcarrier basis. We note that RSM-1 brings gains of $3 \mathrm{~dB}$ and $4 \mathrm{~dB}$ gains respectively for $G_{\mathrm{SR}_{n}} / G_{\mathrm{R}_{n} \mathrm{D}}=10 \mathrm{~dB}$ and $30 \mathrm{~dB}$, respectively (these results are omitted due to space constraints). This demonstrates that having reliable $\mathrm{S} \rightarrow \mathrm{R}$ links becomes a favorable condition for relay selection.

\section{CONCLUSION}

In this paper, we have investigated the performance of a precoded cooperative OFDM system with AF relaying in a multirelay scenario. Through the derivation of PEP, we have identified that the diversity order for the OFDM multi-relay system is worsened by a factor of $(\ln \rho)^{\sum_{n=1}^{N} \min \left(L_{\mathrm{SR}_{n}}+1, L_{\mathrm{R}_{n} \mathrm{D}}+1\right)}$, where channel lengths in underlying source-to- $n^{\text {th }}$ relay, and $n^{\text {th }}$ relay-to-destination are given by $L_{\mathrm{SR}_{n}}+1$ and $L_{\mathrm{R}_{n} \mathrm{D}}+$ 1 ), $n=1, \cdots, N$, respectively. If that factor is ignored, the maximum diversity order for the multi-relay system is $L_{\mathrm{SD}}+1+\sum_{n=1}^{N} \min \left(L_{\mathrm{SR}_{n}}+1, L_{\mathrm{R}_{n} \mathrm{D}}+1\right)$ where $L_{\mathrm{SD}}+1$ is the channel length from the source-to-destination. Based on the PEP expression, we have also proposed two relay selection methods which provide further performance improvements. Finally, we have presented Monte-Carlo simulations to corroborate the analytical results and to provide performance comparisons.

\section{APPENDIX}

Using the result in [17, Eq (4a), page 200], we upper bound the expectation in (6) with respect to $\mathbf{h}_{\mathrm{SD}}$ by

$$
\begin{aligned}
\mathbb{E}_{\mathbf{h}_{\mathrm{SD}}} & {\left[\exp \left(\frac{-\rho \mathbf{h}_{\mathrm{SD}}^{H} \mathbf{U}_{\mathrm{SD}}^{H} \mathbf{U}_{\mathrm{SD}} \mathbf{h}_{\mathrm{SD}}}{8}\right)\right] } \\
\leq & \frac{\left(8 L_{\mathrm{SD}}+8\right)^{L_{\mathrm{SD}}+1}}{\operatorname{det}\left(\mathbf{U}_{\mathrm{SD}}^{H} \mathbf{U}_{\mathrm{SD}}\right)} \rho^{-\left(L_{\mathrm{SD}}+1\right)} .
\end{aligned}
$$

Next, taking expectation with respect to $\mathbf{h}_{\mathrm{SR}_{n}}$, we obtain $J_{n}$ in (6) as

$$
\begin{aligned}
J_{n}=\mathbb{E}_{\mathbf{h}_{R_{n} \mathrm{D}}}[ & \operatorname{det}\left(\mathbf{I}+\frac{\rho G_{\mathrm{SR}_{n}} A_{n}^{2}}{8\left(L_{\mathrm{SR}_{n}}+1\right)} \mathbf{V}_{\mathrm{SR}_{n}}^{H}\left(\mathbf{I}+A_{n}^{2} \mathbf{D}_{\mathrm{R}_{n} \mathrm{D}} \mathbf{D}_{\mathrm{R}_{n} \mathrm{D}}^{H}\right)^{-1}\right. \\
& \left.\left.\times \mathbf{D}_{\mathrm{R}_{n} \mathrm{D}} \mathbf{D}_{\mathrm{R}_{n} \mathrm{D}}^{H} \operatorname{diag}\left(\|\mathbf{u}\|^{2}\right) \mathbf{V}_{\mathrm{SR}_{n}}\right)^{-1}\right]
\end{aligned}
$$

For a given error vector $\mathbf{e}=\mathbf{s}-\mathbf{s}^{\prime} \neq \mathbf{0}$, errors occur in at least one group. The precoded error vector in a group has all nonzero entries if the error vector in that group is non-zero [10]. Suppose the $m^{\text {th }}$ group is one of the erroneous groups, i.e., $\mathbf{s}_{m} \neq \mathbf{s}_{m}^{\prime}, 1 \leq m \leq M$. Then we have $\mathbf{u}_{m}(q) \neq 0, \forall q \in$ $\{1,2, \cdots, L+1\}$. Noting $L=\max \left(L_{\mathrm{SD}}, L_{\mathrm{SR}_{n}}, L_{\mathrm{R}_{n} \mathrm{D}}\right)$ and introducing a matrix $\mathbf{P}$ which contains the first $L_{\mathrm{SR}_{n}}+1$ rows in $\boldsymbol{\Psi}_{m}$, we rewrite (12) as

$J_{n}=\mathbb{E}_{\mathbf{h}_{n} \mathrm{D}}\left[\operatorname{det}\left(\mathbf{I}+\frac{\rho G_{\mathrm{SR}_{n}} A_{n}^{2}}{8\left(L_{\mathrm{SR}_{n}}+1\right)} \mathbf{V}_{\mathrm{SR}_{n}}^{H} \mathbf{P}^{T} \mathbf{P G}_{n} \mathbf{P}^{T} \mathbf{P V}_{\mathrm{SR}_{n}}\right)^{-1}\right]$

where $\mathbf{G}_{n}=\left(\mathbf{I}+A_{n}^{2} \mathbf{D}_{\mathrm{R}_{n} \mathrm{D}} \mathbf{D}_{\mathrm{R}_{n} \mathrm{D}}^{H}\right)^{-1} \mathbf{D}_{\mathrm{R}_{n} \mathrm{D}} \mathbf{D}_{\mathrm{R}_{n} \mathrm{D}}^{H} \operatorname{diag}\left(\|\mathbf{u}\|^{2}\right)$. Since the matrix inside the determinant bracket of (13) is positive definite, an upper bound for $J_{n}$ can be written as

$$
\begin{aligned}
J_{n} \leq & \frac{\left(L_{\mathrm{SR}_{n}}+1\right)^{2\left(L_{\mathrm{SR}_{n}}+1\right)}}{\varpi_{n}} \mathbb{E}_{\mathbf{h}_{\mathrm{R}_{n} \mathrm{D}}}\left[\prod_{\ell=1}^{L_{\mathrm{SR}_{n}}+1} \frac{1}{1+\rho \alpha_{n}\left|\mathbf{u}\left(i_{m, \ell}\right)\right|^{2}}\right. \\
& \left.\times\left(1+\frac{\frac{\rho \alpha_{n}|\mathbf{u}(i)|^{2}}{A_{n}^{2}+\rho \alpha_{n}\left|\mathbf{u}\left(i_{m, \ell}\right)\right|^{2} A_{n}^{2}}}{\left|\mathbf{D}_{\mathrm{R}_{n} \mathrm{D}}\left(i_{m, \ell}\right)\right|^{2}+\frac{1}{A_{n}^{2}+\rho \alpha_{n}\left|\mathbf{u}\left(i_{m, \ell}\right)\right|^{2} A_{n}^{2}}}\right)\right]
\end{aligned}
$$

where $\alpha_{n}=\left(L_{\mathrm{SR}_{n}}+1\right) G_{\mathrm{SR}_{n}} / 8$, and $i_{m, \ell}=(\ell-1) M+$ $m, \quad \ell=1, \cdots, L_{\mathrm{SR}_{n}}+1$. Since the components in $\mathbf{h}_{\mathrm{R}_{n} \mathrm{D}}$ are i.i.d. Gaussian with variance $1 /\left(L_{\mathrm{R}_{n} \mathrm{D}}+1\right)$, we have $\mathbf{D}_{\mathrm{R}_{n} \mathrm{D}}\left(i_{m, \ell}\right) \sim \mathcal{C N}(0,1)$. Taking the expectation with respect to $\mathbf{h}_{\mathrm{R}_{n} \mathrm{D}}$, we obtain the following two cases at high SNR:

Case $1\left(L_{\mathrm{SR}_{n}} \leq L_{\mathrm{R}_{n} \mathrm{D}}\right)$ :

$J_{n \text { case } 1} \leq a_{n \text { case } 1}\left(\rho^{-1} \ln \rho\right)^{L_{\mathrm{SR}_{n}}+1}+O\left(\rho^{-\left(L_{\mathrm{SR}_{n}}+1\right)}(\ln \rho)^{L_{\mathrm{SR}_{n}}}\right)$

where $a_{n \text { casel }}=\frac{\left(8 \pi\left(L_{\mathrm{SR}_{n}}+1\right)\right)^{\left(L_{\mathrm{SR}_{n}}+1\right)}}{\varpi_{n}\left(v G_{\mathrm{R}_{n} \mathrm{D}}\right)^{L_{\mathrm{SR}_{n}}+1}}$. Here, we have used the fact $\left|\mathbf{u}\left(i_{m, \ell}\right)\right|^{2} \geq v[10]$.

Case $2\left(L_{\mathrm{SR}_{n}}>L_{\mathrm{R}_{n} \mathrm{D}}\right)$ :

$J_{n \text { case } 2} \leq a_{n \text { case } 2}\left(\rho^{-1} \ln \rho\right)^{L_{\mathrm{R}_{n} \mathrm{D}}+1}+O\left(\rho^{-\left(L_{\mathrm{R}_{n} \mathrm{D}}+1\right)}(\ln \rho)^{L_{\mathrm{R}_{n} \mathrm{D}}}\right)$

where $a_{n \text { case } 2}=\frac{\left(8 \pi\left(L_{\mathrm{SR}_{n}}+1\right)\right)^{L_{\mathrm{R}_{n} \mathrm{D}}+1}}{\varpi_{n}\left(v G_{\mathrm{R}_{n} \mathrm{D}}\right)^{L_{\mathrm{R}_{n} \mathrm{D}} \mathrm{D}^{+1}}}$.

An upper bound of $J_{n}$ for both cases can be defined as,

$J_{n} \leq \frac{\left(8 \pi\left(L_{\mathrm{SR}_{n}}+1\right)\right)^{k_{n}}}{\varpi_{n}\left(v G_{\mathrm{R}_{n} \mathrm{D}}\right)^{k_{n}}}\left(\rho^{-1} \ln \rho\right)^{k_{n}}+O\left(\rho^{-k_{n}}(\ln \rho)^{k_{n}-1}\right)$

Replacing (14) and (11) in (6), we obtain (8).

\section{REFERENCES}

[1] J. N. Laneman, D. N. C. Tse, and G. W. Wornell, "Cooperative diversity in wireless networks: efficient protocols and outage behavior," IEEE Trans. Inform. Theory, vol. 49, pp. 3062-3080, Dec. 2004.

[2] H. Ochiai, P. Mitran, and V. Tarokh, "Variable-rate two-phase collaborative communication protocols for wireless networks," IEEE Trans. Inform. Theory, vol. 52, no. 9, pp. 4299-4313, Sept. 2006.

[3] G. Li and H. Liu, "On the capacity of the broadband relay networks," in Proc. Asilomar Conf. Signals, Syst., Comput., Nov. 2004, pp. 1318-1322.

[4] M. Herdin, "A chunk based OFDM amplify-and-forward relaying scheme for $4 \mathrm{G}$ mobile radio systems," in Proc. IEEE Int. Conf. Commun. (ICC), June 2006, pp. 4507-4512.

[5] W. P. Siriwongpairat, A. K. Sadek, and K. J. R. Liu, "Cooperative communications protocol for multiuser OFDM networks," IEEE Trans. Wireless Commun., vol. 7, no. 7, pp. 2430-2435, July 2008.

[6] L. Dai, B. Gui, and L. J. Cimini, "Selective relaying in OFDM multihop cooperative networks," in Proc. IEEE Wireless Commun. Networking Conf., Mar. 2007, pp. 963-968.

[7] O.-S. Shin, A. Chan, H. T. Kung, and V. Tarokh, "Design of an OFDM co-operative diversity system," IEEE Trans. Veh. Technol., vol. 56, no. 4, pp. 2203-2215, July 2007.

[8] S. Barbarossa and G. Scutari, "Distributed space-time coding for regenerative relay networks," IEEE Trans. Wireless Commun., vol. 4, no. 5, pp. 2387-2399, Sept. 2005.

[9] H. Mheidat, M. Uysal, and N. Al-Dhahir, "Equalization techniques for distributed space-time block codes with amplify-and-forward relaying," IEEE Trans. Signal Processing, vol. 55, no. 5, pp. 1839-1852, May 2007.

[10] Z. Liu, Y. Xin, and G. B. Giannakis, "Linear constellation precoding for OFDM with maximum multipath diversity and coding gains," IEEE Trans. Signal Processing, vol. 51, no. 3, pp. 416-427, Mar. 2004.

[11] J. W. Craig, "A new, simple, and exact result for calculating the probability of error for two-dimensional signal constellations," in Proc. IEEE Milit. Commun. Conf., Mclean, VA, Oct. 1991, pp. 571-575. 
[12] Y. Ding, J. K. Zhang, and K. M. Wong, "The amplify-and-forward half-duplex cooperative system: pairwise error probability and precoder design," IEEE Trans. Signal Processing, vol. 55, no. 2, pp. 605-617, Feb. 2007.

[13] Y. Jing and B. Hassibi, "Distributed space-time coding in wireless relay networks," IEEE Trans. Wireless Commun., vol. 5, no. 12, pp. 35243536, Dec. 2006.

[14] M. Uysal, "Diversity analysis of space-time coding in cascaded Rayleigh fading channels," IEEE Commun. Lett, vol. 10, no. 3, pp. 165-167, Mar. 2006.
[15] A. Hottinen and T. Heikkinen, "Optimal subchannel assignment in a two-hop OFDM relay," in Proc. IEEE Signal Proc. Wkshp. Signal Advances Wireless Commun. (SPAWC), June 2007, pp. 1-5.

[16] Y. Ding and M. Uysal, "Multi-relay cooperative OFDM with amplifyand-forward relaying," in Proc. IEEE Radio Wireless Symp. (RWS), San Diego, CA, Jan. 2009 (invited paper).

[17] G. L. Turin, "The characteristic function of Hermitian quadratic forms in complex normal random variables," Biometrika, pp. 199-201, June 1960. 